\title{
San Antonio 2016: Progress on the Long Way to Individualized Treatment of Breast Cancer
}

\author{
Chair: \\ Volkmar Müllera \\ Participants: Oleg Gluz ${ }^{b} \quad$ Jean-Yves Pierga ${ }^{c} \quad$ Marc Thill $^{d} \quad$ Brigitte Rack $^{\mathrm{e}} \quad$ Isabell Witzel ${ }^{f}$ \\ ${ }^{a}$ Konservative gynäkologische Onkologie, Klinik und Poliklinik für Gynäkologie, Universitätsklinikum Hamburg-Eppendorf, Hamburg, Germany; \\ ${ }^{b}$ Evangelisches Krankenhaus Bethesda, Mönchengladbach, Germany; \\ 'Institut Curie, Paris, France; \\ ${ }^{\mathrm{d}}$ Ludwig-Maximilians-Universität, Klinik und Poliklinik für Frauenheilkunde und Geburtshilfe, Klinikum Innenstadt, Munich, Germany; \\ ${ }^{e}$ Klinik für Gynäkologie und Geburtshilfe, Agaplesion Markus Krankenhaus, Frankfurt/M., Germany; \\ ${ }^{f}$ Brustzentrum am UKE, Kompetenzzentrum für familiären Brust- und Eierstockkrebs, Klinik und Poliklinik für Gynäkologie, Universitätsk- \\ linikum Hamburg-Eppendorf, Hamburg, Germany
}

The annual San Antonio Breast Cancer Symposium is the most important breast cancer conference. Each year, clinical studies and basic research results are presented. This leads to a unique atmosphere of exchange between basic researchers and clinicians. Here, experts from both fields of research describe their personal highlights from the meeting in December 2016.

Volkmar Müller

\section{Question 1: Breast Cancer Surgery: Did You See New Findings with Clinical Implications?}

Pierga: There were very few new findings in surgery during this meeting. I would highlight the GANEA 2 trial presented by Classe et al. that raised the question of avoiding axillary dissection in selected patients after neoadjuvant chemotherapy (NACT). Of 590 patients who were considered as N0 before NACT, defined by the absence of involved nodes at axillary ultrasound and/or by cytology, 418 had a negative sentinel lymph node biopsy (SLNB) and no further axillary treatment. After a median follow-up of 3 years, only 1 axillary relapse has occurred (0.2\%). Despite this short follow-up for analysis of loco-regional relapse, this trial tends to show there is little risk in avoiding large surgery in N0 patients after NACT. The strategy in patients with involved node before NACT remains to be defined.

Gluz: At least 1 important surgery study has been presented at the SABCS. The GANEA 2 trial investigated survival effect of SLNB after completion in patients with clinically node-negative $(\mathrm{N}-)$ tumors prior to neoadjuvant chemotherapy. A low false-neg- ative rate of such procedures was reported by several trials and led to the guidelines recommendation of allowing SLNB after NACT together with surgery, although the survival effect of this timing was unclear. A very high SLN identification rate of $97 \%$ in 518 patients has been shown in the trial. 418 patients ( $97 \%$ of all patients with negative SLN) were treated by SLNB alone. Only 1 axillary relapse and a 3-year disease-free survival (DFS) of $94.8 \%$ were observed after 3 years of median follow-up. These data confirm the oncological safety of SLNB timing in breast cancer patients.

Rack: The GANEA 2 trial (Classe et al.) prospectively evaluated the role of SLNB as only surgical treatment to the axilla in cN0 patients after NACT. During follow-up, the authors observed only 1 axillary relapse in 418 patients. Despite a short median follow-up of 36 months these data confirm the safety of this procedure and support this years' AGO guidelines rating SLNB after neoadjuvant chemotherapy with ' + '.

Thill: The only practice-changing or recommendation-confirming trial was the GANEA 2 trial. In the ACOSOG Z1071 trial the false-negative rate of initially node-positive patients was $21 \%$, in the SENTINA trial it was $18.5 \%$. That is the reason why we currently not recommend treating this group of patients with a SLNB. This is the background for the GANEA 2 trial, a prospective, multi-institutional, 2-arm, French cohort study, that compared N- and nodepositive $(\mathrm{N}+)$ patients after NACT. The initially $\mathrm{N}$ - patients underwent SLNB +/- axillary lymph node dissection (ALND) (cohort 2) and the N+ cohort received SLNB + ALND (cohort 1). The SLN identification rate in cohort 1 was $79.8 \%$ and in cohort $297.0 \%$, respectively. The false-negative rate in cohort 1 was $12 \%$. These re-

\section{KARGER}

(๑) 2017 S. Karger GmbH, Freiburg

Fax +497614520714
Prof. Dr. med. Volkmar Müller 
sults do not lead to a change of our current recommendation, not to perform SLNB in initially $\mathrm{N}+$ early breast cancer patients. A falsenegative rate of $>20 \%$ is definitely too high and SLNB has not been proven to be a safe procedure outside trials. However, for initially $\mathrm{N}$ - patients, SLNB after NACT is a safe procedure, as only $1 / 418$ axillary relapse occurred. Axillary status has to be proven with ultrasound and if necessary with core needle or fine needle biopsy. The SenTa trial that will start in the second quarter of 2017 will evaluate more intensively the concordance between the biopsied positive lymph node and the SLN using clip marking.

Witzel: The GANEA 2 trial showed that in the neoadjuvant setting it is safe to spare pre-neoadjuvant sentinel dissection if the nodes are not initially involved. However, in the group of patients with $\mathrm{N}+$ disease at initial diagnosis, a false-negative rate of $>20 \%$ after neoadjuvant chemotherapy implies that axillary dissection after neoadjuvant chemotherapy still has to be performed in this group of patients even if their nodes responded to chemotherapy.

In the British POSH trial, breast cancer patients younger than 40 years were analyzed. BRCA status was not associated with an impaired prognosis. In triple-negative breast cancer (TNBC) BRCA mutation was even associated with an $11 \%$ improved 10 year survival rate and no advantage could be seen with regard to survival for patients in whom a bilateral mastectomy was performed. This trial helps to counsel young patients with breast cancer that a more extensive surgery of the breasts does not necessarily result in better survival rates.

\section{Question 2: Triple Negative Breast Cancer: Did You Learn New Aspects for the Treatment that Might Lead to Progress in the Clinical Management?}

Gluz: Patel et al. presented data from the National Cancer Database on 13,065 patients with pT1a, $1 \mathrm{~b}$ and $1 \mathrm{c}$ node-negative tumors treated by adjuvant chemotherapy or not. With all caveats of registry data (e.g. patients treated by chemotherapy were younger etc.) a significant benefit of chemotherapy was observed in pT1b and pT1c tumors, and only a non-significant trend to better 4-year overall survival (OS) (94 vs. 98\%) in pTla tumors. In absence of randomized trials in this cohort, these registry data are very important for clinical routine.

Pierga: No significant modification for the treatment of TNBC has been presented during this meeting. New results of the TNT trial comparing docetaxel to carboplatin in first line for metastatic TNBC presented by Andrew Tutt, revealed that a methylation profile of the BRCA1 gene has no value to predict response to platinum. Only germline mutations of $B R C A 1$ were predictive of response to carboplatin. Brocade 3 , a phase II randomized trial in $B R C A 1 / 2$ mutation carriers, failed to show a benefit of the addition of veliparib, a PARP inhibitor to a combination of carboplatin and paclitaxel. An interesting poster on the national data in the US re- ported an equivalent $\mathrm{OS}$ in the population of 15,000 TNBC patients receiving adjuvant and neoadjuvant chemotherapy. Despite adjustment on demographics, tumor, and treatment factors, bias due to the absence of randomization should be kept in mind to interpret these results.

Rack: There were interesting presentations not directly regarding TNBC, but hereditary breast cancer. Couch et al. applied gene sequencing to a large cohort of 60,000 breast cancer patients in order to identify mutations in predisposition genes for breast cancer. Several genes were identified and rated according to their risk for breast cancer development. These data will help the detection of germline mutations and to advise patients with a family history of breast cancer. Eccles et al. investigated the role of $B R C A 1 / 2 \mathrm{mu}-$ tations for the further course of the disease. In the POSH trial 3,053 young breast cancer patients ( $\leq 40$ years) were included. $B R C A$ mutations did not affect survival compared to patients without these mutations, both for the whole cohort as well as for TNBC patients. Especially important in my view was that TNBC patients with BRCA1/2 mutations did not benefit from contralateral mastectomy. Therefore, these women should not be advised to undertake this very invasive procedure.

Thill: Unfortunately, I missed convincing study results with checkpoint inhibitors or PARP inhibitors. Carsten Denkert gave a very nice talk about tumor infiltrating lymphocytes (TILs) based on a meta-analysis of 3,771 patients. He confirmed that in TNBC TILs are a predictive and prognostic factor. High TILs are associated with a higher pathological complete response (pCR) and a better prognosis. This suggests that TNBC is an immunogenic subtype that offers new treatment options.

Data of PARP inhibitor studies are urgently awaited, therefore I was glad that the results of the BROCADE phase II trial, comparing carboplatin and paclitaxel +/- veliparib in BRCA1/2 locally recurrent or metastatic breast cancer patients (hormone receptor (HR) positive and negative), were presented by Han and co-authors. However, the results were unsatisfactory, as progression-free survival (PFS) only increased by 1.8 months (12.3 vs. 14.1 months, $\mathrm{p}=0.231$ ) and OS only by 2.4 months ( 25.9 vs. 28.3 months, $\mathrm{p}=$ $0.157)$. Nevertheless, a significant difference in overall response rate (ORR) was shown (61.3 vs. $77.8 \%, \mathrm{p}=0.027)$. It seems that we have to wait longer for better results - anyway, many trials are ongoing at the moment.

Witzel: A meta-analysis by Natori et al. looked at the addition of capecitabine to standard chemotherapy in early breast cancer. 8 randomized controlled studies with 9,302 patients could be included. Although there was no benefit with regard to survival in unselected cases, the addition of capecitabine to standard chemotherapy significantly improved DFS in TNBC vs. non-TNBC (HR $=0.72$ vs. $1.01, \mathrm{p}=0.02$ ). The benefits had to be paid with higher toxicities (diarrhea and hand foot syndrome). The authors concluded that in selected patients with TNBC the addition of capecitabine could be discussed. 


\section{Question 3: Did You See New Developments in Targeted Therapies of Breast Cancer Patients that Will Find Their Way into Practice?}

Gluz: The PrE0102study presented by Kornblum et al. investigated the effect of fulvestrant $+/$ - everolimus in 131 patients resistant to aromatase inhibitors (AI) in the metastatic setting. In concordance with the BOLERO II (exemestane+/- everolimus) or PALOMA 3 (fulvestrant +/- the CDK 4/6 inhibitor palbociclib) trials the median PFS has been extended from 5.1 to 10.4 months in favor of combination therapy, however, at the cost of increased toxicity (grade 3 events 48 vs. 14\%). No OS effect has been observed yet in this rather small study. This combination, not yet approved of, extends a field of possible combinations in metastatic treatments.

Prat et al. presented data from the PAMELA study investigating a neoadjuvant double HER2 blockade by trastuzumab + lapatinib without chemotherapy (+ endocrine therapy in HR+/HER2+ disease) given for 18 weeks in 150 patients. Only $67 \%$ of samples have shown the HER2-enriched subtype by PAM-50. In the HER2 enriched subtype a pCR of $40.6 \%$ was observed (vs. only $10 \%$ in other subtypes) and $43 \%$ in the HR-/HER2+ disease (where most tumors were HER2-enriched by PAM-50). Yet, if combination with chemotherapy would lead to a higher pCR of about $70-80 \%$ in these patients, it remains unclear whether this chemotherapy-free option, associated with favorable side effects profile, may not be used in some selected patients with HER2+ disease (e.g. fragile patients).

Pierga: The PrE0102 trial (Kornblum et al.) has shown that the combination of fulvestrant + everolimus was superior to fulvestrant alone in $\mathrm{HR}+$ metastatic breast cancer (MBC) patients. The difference for PFS (10 vs. 5 months) was superimposable to the difference reported in the BOLERO2 trial with exemestane. Fulvestrant could be an option for the combination with an anti-mTOR agent like in the TAMRAD trial with tamoxifen.

The BELLE3 trial (Di Leo et al.) has shown the superiority of a combination of PI3KCA inhibitor (buparlisib) + fulvestrant to fulvestrant alone in PFS (4 vs. 2 months). Despite a statistically significant difference, the development of nonspecific anti-PI3KCA inhibitors seems compromised by toxicity, particularly depression and risk of suicide. It is unlikely that this early generation of this type of drug will find its way into clinical practice.

Rack: In the PERTAIN study postmenopausal HR+HER2+ patients without previous treatment of chemotherapy for advanced disease were treated with an AI in combination with dual blockade (trastuzumab + pertuzumab) vs. AI + trastuzumab. The dual blockade was associated with a significantly better PFS (15.8 vs. 18.8 months; $\mathrm{p}=0,007)$. This regimen could therefore help to postpone chemotherapy treatment for another treatment line, especially due to its excellent tolerability.

One oral (Hurvitz) and one poster presentation (Gianni) showed interesting preliminary data on CDK4/6 inhibitors in the neoadjuvant setting. The authors could show an impressive decline of Ki67
2 weeks after the start of treatment in most patients. Therefore, this chemotherapy-free treatment should be further evaluated.

Thill: At the moment, CDK 4/6 inhibition is a new milestone in the treatment of metastatic HR+ breast cancer and the most promising approach for the treatment of $\mathrm{HR}+$ early breast cancer. Besides excellent data of the PALOMA 1-3 trials and the MONALEESA- 2 trial that were presented and published already, Hurvitz et al. presented very nice data of the phase II neoMONARCH study. In this study, the CDK 4/6 inhibitor abemaciclib was offered to postmenopausal HR+ patients with early breast cancer in a window of opportunity manner. The patients received abemacilib alone vs. abemaciclib + anastrozole vs. anastrozole alone for 14 days. Afterwards a re-biopsy was taken and all patients received the combination of abemaciclib + anastrozole for 14 weeks. The results were impressive. After 2 weeks the Ki67 dropped significantly in both abemaciclib arms, induced a profound cell cycle arrest and, moreover, the majority of patients who received abemaciclib + anastrozole received an objective tumor response after 14 weeks of treatment. I think that these results have to lead to a broad testing of CDK 4/6 inhibition in early breast cancer, fortunately studies are already ongoing.

Another promising target is PI3K and some efficacy results of PI3K inhibitors were presented at the congress. After unsatisfactory results of the pan-PI3K inhibitor pictilisib last year the BELLE-3 phase-III study was presented. Fulvestrant + /- buparlisib (a pan-PI3K inhibitor) was evaluated in postmenopausal AI pretreated patients with locally advanced or metastatic breast cancer. The authors showed a significant difference in PFS (3.9 vs. 1.8 months; $\mathrm{p}<0.001$ ), especially in patients with visceral disease. No benefit was shown for patients with non-visceral disease. However, in the experimental arm toxicity significantly increased. Therefore, I would like to conclude that we have to focus on specific PI3K inhibitors like taselisib or alpelisib as they have lower toxicity.

Witzel: Combination therapies between different strategies (e.g., CDK 4/6 inhibitors in combination with PIK3CA inhibitors and endocrine therapy) offer the opportunity to overcome endocrine resistance and to prolong response rates until the initiation of chemotherapy in patients with HR+ metastatic breast cancer. Some of these combinations will find their way into clinical practice but up to now, we do not know which ones that will be.

\section{Question 4: Which of the Presented Data on Adjuvant and Neoadjuvant Chemotherapy Will Be Applied in Your Clinical Routine?}

Gluz: A Danish study group presented data from a randomized trial that compared 6 cycles of docetaxel/cyclophosphamide to $3 \mathrm{cy}$ cles of epirubicin/cyclophosphamide/5-fluoruracil followed by $3 \mathrm{cy}$ cles of docetaxel in 2,012 node-positive or high-risk node-positive patients with normal topoisomerase-II-alpha (topo-II- $\alpha$ ) tumors, defined as FISH ratio between 0.8 and 1.9. The rationale is based on 
the observation, that $6 \times \mathrm{CEF}$ has been shown to be superior to CMF only in cases with topo-II- $\alpha$-amplified or -deleted tumors by retrospective analysis from DBCG-89G trial. Although topo-II- $\alpha$ amplification is often associated with HER2 amplification/overexpression, $11 \%$ of patients in the trial had HER2+ tumors. After median followup of 5 years, no significant difference in terms of DFS and OS has been observed between both study arms, but anthracycline-free regimens show a more favorable toxicity profile.

Interestingly, these data correlate very well with a smaller study from The Netherlands that included 662 patients with high-risk N0 or node-positive HER2- disease. In this study no significant efficacy difference has been observed between adjuvant chemotherapy by $6 \times$ TAC or 6 cycles of dose-dense EC. However, very similarly to the first pooled analysis data from the $\mathrm{ABC}$ trials presented by Blum et al., a trend toward higher efficacy of anthracycline containing chemotherapy has been observed in TNBC.

Pierga: Three randomized trials evaluated the extension of adjuvant hormonal treatment with AI after 5 years of standard treatment. All these trials were negative for their primary endpoint, which was DFS. The main trial, the NSABP B42 presented by Mamounas et al., included around 4,000 patients and showed a trend for a decrease of distant metastases. Another trial, DATA, in 1,912 patients showed a trend for a benefit in patients with larger tumor and nodal involvement. The conclusion of these results in clinical practice is that there is no absolute demonstration of a benefit of prolongation of hormonal treatment with AI. There could be an advantage for patients with higher risk of relapse (larger tumor, $\mathrm{pN}+$ ). The decision for prolonging AI should be discussed individually with the patients, considering possible side effects as osteoporosis and possible arterial vascular toxicity of AI.

Rack: The READ trial showed no difference between 3EC-3D and $6 \mathrm{DC}$ in topo-II- $\alpha$-normal patients. This regimen is an option for patients who have contraindications against anthracyclines.

Thill: No new data on neoadjuvant or adjuvant chemotherapy were shown that will influence my clinical routine. However, important data clarifying the value of an extended anti-hormonal treatment were presented. Since the ATLAS and the aTTom trials, extended anti-hormonal treatment (EAT) is under intensive discussion. Moreover, since the ASCO meeting in June 2016 with the EBCTCG presentation and the MA.17R study the discussion is even more intensive. In San Antonio, 3 other studies evaluating EAT were presented: i) the multicenter phase III DATA study comparing 3 vs. 6 years of anastrozole after 2-3 years of tamoxifen in postmenopausal women with $\mathrm{HR}+$ early breast cancer, ii) the phase III IDEAL trial with an extended letrozole treatment after 5 years of adjuvant endocrine therapy, and iii) the phase III NSABP B-42 trial to evaluate EAT (5 years of letrozole) in postmenopausal women with HR+ breast cancer who have completed previous adjuvant endocrine therapy. To make a long story short, the results of the 3 studies did not support EAT. In the DATA trial, only chemo-treated patients with $\mathrm{ER}+$ and $\mathrm{PR}+$ and $\mathrm{N}+$ dis- ease benefited, in the IDEAL trial no significant differences in disease-related outcomes were noticed for an extended AI treatment longer than 2.5 years, and in the NSABP B- 42 the extended $\mathrm{AI}$ application resulted only in a significant benefit in breast cancer free interval (BCFI) and distant recurrence (DR), but not in OS. Therefore, I agree with the conclusion of the disputant Michael Gnant and would recommend an extend AI application of 2.5 years after $2-5$ years of tamoxifen. For a patient after AI we have to decide carefully and under consideration of toxicity.

Witzel: I found the concept of tumor-infiltrating lymphocytes (TILs) very interesting. Carsten Denkert presented a meta-analysis of 3,771 patients who had received neoadjuvant chemotherapy. Here, higher TILs were associated with better prognosis in triplenegative and in HER2+ patients, whereas the relationship was opposite in luminal tumors. Thus, high TILs are a strong predictive factor in all subtypes of breast cancer but are only prognostic in TNBC and HER2+ subtypes. In the luminal subtype, studies focusing on the role of TILs in resistance to endocrine therapy are missing.

\section{Question 5: 'Trials in Progress' Session: What Are Important Breast Cancer Trials Currently Recruiting?}

Gluz: The German INSEMA trial, investigating the need of axillary intervention in patients with clinically node-negative breast cancer, seems to be a most interesting currently ongoing clinical trial, which can immediately impact clinical practice in thousands of patients. 1,641 patients have been recruited into the trial by 136 centers in Germany and Austria.

Pierga: Inhibitors of cyclin-dependent kinases (CDK), such as palbociclib or ribociclib have demonstrated their interest in first line treatment combined with hormonal treatment in HR+ HER2MBC patients. Their comparison with chemotherapy, such as capecitabine is currently evaluated in the PEARL study run by the GEICAM group (OT2-01-06).

The TRANSCAN JTC2011 trial is currently evaluating the use of the FES PET Scan (with labeled estradiol) to predict response to a first line of hormonal treatment in HR+ HER2- MBC patients. In patients with strong binding of the tracer, endocrine treatment is prescribed. In patients with low fixation, chemotherapy or endocrine treatment are randomized. This could help to define the best treatment strategy in this population (OT3-03-03).

Thill: From a German view, the DETECT V trial is very important, as it is, to my knowledge, the only trial that evaluates a circulating tumor cell (CTC)-dependent treatment decision in MBC. Another very important trial is the INSEMA study, answering the question: can we avoid SLNB in N- early breast cancer patients?

In general, studies that evaluate checkpoint-inhibitors are important, as we have to find the most beneficial treatment approach 
for the most responding patient subgroup, especially, we have to identify the long-time responders and their predictive factors. Moreover, I really would like to know, how beneficial PARP inhibitors are.

Rack: I agree, DETECT and INSEMA are important trials.

Witzel: I found that one of the most interesting, currently recruiting trials for breast cancer surgeons was the LORIS trial run in the UK, in which patients with a centrally confirmed diagnosis of low-grade DCIS are randomized to receive either standard treatment or active monitoring with annual mammography and are followed up for at least 10 years. This trial really focuses on a patient group that might be overdiagnosed in the mammography-screened population and will give an answer whether low-grade DCIS always has to be treated.

\section{Question 6: Did You Find Further Aspects of Relevance?}

Gluz: Hurvitz et al. presented the NeoMonarch study, investigating a possible proliferation effect of anastrozole, anastrozol + CDK 4/6 inhibitor abemaciclib or abemaciclib alone after 2 weeks of treatment. This study has shown a stronger proliferation decrease by combination therapy vs. anastrozole alone. Interestingly, no difference has been found between monotherapy with abemaciclib and combination therapy (mean Ki-67 decrease of 90\%). Only the combination therapy has been continued for further 16 weeks, hence no clinical response data on monotherapy will be available from this study.

Pierga: The IMENEO meta-analysis presented by Bidard et al. has gathered data on more than 2,000 patients who received NACT and had circulating tumor cell (CTC) detection. This large series has shown that CTC detection is independent of tumor characteristics and of pCR. CTC are a very strong prognostic factor for OS, at baseline or after NACT. However, this prognostic factor has not yet demonstrated its clinical utility and clinical trials based on this biomarker to decide on adjuvant treatment prescription are still needed.

Rack: Prolonged endocrine treatment with AI (DATA, IDEAL, NSABP-B42): treatment should be discussed depending on side effects and risk profile. I was also impressed by the CTC meta-analysis in neoadjuvant $\mathrm{BC}$, presented by Bidard et al.

Thill: I would like to mention the phase II PERTAIN study. This study examined the value of adding pertuzumab to trastuzumab + AI in postmenopausal, HER2+, HR+, locally advanced or metastatic breast cancer patients and met its clinical endpoint. The in dual blockade by addition of pertuzumab led to a significant difference in PFS of 3.09 months (18.89 vs. 15.80 months, HR 0.65).
Now we know that a dual blockade $+\mathrm{AI}$ is more beneficial than trastuzumab + AI alone, but we still do not know whether we need an AI added to dual blockade, as in the study the dual blocked-only arm was missing. Therefore, this trial has no consequences for my daily work. Taxane + pertuzmab + trastuzumab is my first-line standard treatment. Anyway, an endocrine treatment plus dual blockade as first-line therapy is not approved.

Witzel: Ivana Sestak presented a further analysis of the transATAC trial comparing several gene signatures and a clinical assessment score separately for node-negative $(\mathrm{n}=591)$ and node-positive $(n=227)$ patients that had received endocrine therapy in the ATAC trial. She could show that scores that implement clinical features, such as the ROR score or EpClin, perform better in the node-positive cohort. This presentation will lead to a new extensive discussion about which of the commercially available tests might best predict chemotherapy benefit in breast cancer patients. One has to keep in mind that in this analysis tumor samples of less than $10 \%$ of the initially treated study cohort could be evaluated retrospectively and that the cohort of patients did not receive chemotherapy and was relatively old (median age of 63 in the node-negative and 67 years in the node-positive cohort).

\section{Participants}

Dr. Oleg Gluz

Evangelisches Krankenhaus

Bethesda Mönchengladbach $\mathrm{GmbH}$

Ludwig-Weber-Straße 15, 41061 Mönchengladbach, Germany

oleg.gluz@wsg-online.com

Prof. Jean-Yves Pierga

Institut Curie

26 Rue d'Ulm, 75005 Paris, France

France jean-yves.pierga@curie.fr

PD Dr. med. Brigitte Rack

Klinik und Poliklinik für Frauenheilkunde und Geburtshilfe,

Klinikum Innenstadt

Ludwig-Maximilians-Universität

Maistrasse 11, 80337 Munich, Germany

Brigitte.Rack@med.uni-muenchen.de

PD Dr. med. Marc Thill

Klinik für Gynäkologie und Geburtshilfe

Agaplesion Markus Krankenhaus

Wilhelm-Epstein-Str. 4, 60431 Frankfurt/M., Germany

marc.thill@fdk.info

PD Dr. med. Isabell Witzel, MPH

Brustzentrum am UKE

Kompetenzzentrum für familiären Brust- und Eierstockkrebs

Klinik und Poliklinik für Gynäkologie

Universitätsklinikum Hamburg-Eppendorf

Martinistraße 52, 20246 Hamburg, Germany

iwitzel@uke.de 